\title{
Mechanism of Action of Nicotiflorin from Tricyrtis maculata in the Treatment of Acute Myocardial Infarction: From Network Pharmacology to Experimental Pharmacology
}

\author{
Shangshang $\mathrm{Yu}^{\mathrm{l}, *}$ \\ Qi Guol,* \\ Tianqian Jia ${ }^{2, *}$ \\ Xiaofei Zhang' \\ Dongyan Guo' \\ Yanzhuo Jia' \\ Jia Li $\mathbb{D}^{\prime}$ \\ Jing Sun'
}

'Shaanxi University of Chinese Medicine, Xianyang, People's Republic of China;

${ }^{2}$ Haojing College of Shaanxi University of Science \& Technology, Shaanxi, Xi'an,

People's Republic of China

*These authors contributed equally to this work
Correspondence: Jing Sun

Tel +86 029-38184958

Email 3332606446@qq.com
Purpose: Acute myocardial infarction (AMI) is a cardiovascular disease with a high fatality rate. In this study, we combined network pharmacology and experimental pharmacology and discovered the potential mechanism of action and the active ingredients of the lily, Tricyrtis maculata was discovered. The monomer compound with stronger activity was discovered through in vitro cell experiments.

Methods: Forty known compounds were isolated from T. maculata. Using TCMSP, Swiss Target Prediction, metaTarFisher, GeneCards and OMIM databases, targets of drug compositions and AMI-related genes were obtained, and the differential expression genes between AMI and normal tissues were extracted through the GEO database. Then, through an online mapping tool, the intersection genes were obtained to predict the possible effective components of T. maculata that can be used to treat AMI. The top five targets were selected for molecular docking via the protein-protein interaction (PPI) network to verify the binding activity between key compounds and target proteins. GO and KEGG enrichment analyses of the intersection genes were carried out with the program $\mathrm{R}$ to further screen key genes and effective compositions. On this basis, the compound with more optimal activity was screened and validated in vitro.

Results: In this study, 40 known monomer components were selected, and 1112 predicted genes, 1655 disease genes, 1425 differentially expressed genes, 1206 GO functions and 127 KEGG pathways were obtained. The results of molecular docking showed that the binding of MMP9 with drug components is stable. Through the comprehensive research of network pharmacology and experimental pharmacology, it was shown that T. maculata intervenes in the process of AMI through multicomponent, multitarget, and multichannel synergistic effects. It is speculated that the anti-AMI effect may be related to the regulation of the $\mathrm{Akt} / \mathrm{FoxO} / \mathrm{BCl}$ signaling pathway. Cellular experiments showed that nicotiflorin has satisfactory anti-inflammatory activity and endothelial protection and can reduce the release of nitric oxide (NO), an inflammatory medium after endothelial cell damage.

Conclusion: This study reveals the therapeutic effect and relative mechanism of extract of T. maculata extract on AMI. Analysis revealed that nicotiflorin from T. maculata is a compound with satisfactory anti-inflammatory activity and endothelial protection, which provides a new direction and treatment basis for further experimental exploration and clinical treatment.

Keywords: Tricyrtis maculata, nicotiflorin, network pharmacology, experimental pharmacology, GEO data mining 


\section{Introduction}

Acute myocardial infarction (AMI) is a common cardiovascular disease. This occurs when circulation to a region of the heart is obstructed and myocardial tissue necrosis occurs, and is usually due to thrombosis of an atherosclerotic coronary artery. With risk factors, such as hypertension, abnormal blood lipid, diabetes and smoking continuously increasing in prevalence, the incidence of AMI has also been increasing year by year, posing a severe threat to the health and quality life of patients.

AMI is currently treated by ischemic myocardium reperfusion to save dying heart muscle, drugs used to treat AMI include thrombolytic and antiplatelet agents, anticoagulants, nitrates and calcium ion receptor blockers. However, their efficacy requires further improvement as they are associated with side effects such as headaches, decreased blood pressure, gastrointestinal diseases, impaired liver and kidney function and electrolyte disturbances. Despite great progress in modern medical diagnostic and treatment techniques and in the research and development of drugs for AMI, it remains an incurable disease due to its complex pathogenesis and lack of safe and effective drugs.

Restoration of ischemic myocardium blood perfusion is the main principle of AMI therapy at present. Currently, we mainly rescue the dying myocardium by reperfusion therapy and drug therapy, but these treatments have certain restenosis rate and often have side effects. Protection of myocardial ischemic injury by traditional Chinese medicine (TCM) is a hot research topic at present. ${ }^{1}$ Combined with western medicine, the method of replenishing qi and invigorating blood circulation can significantly reduce the incidence of cardiovascular events in patients.

There has been a great deal of research on using TCM to protect against myocardial ischemic injury. Combined with western medicine, the method of replenishing qi and invigorating blood circulation can significantly reduce the incidence of cardiovascular events in patients. TCM has recently been more closely examined to gain a greater understanding of its complex systems of multicomponent, multiaction targets, and multipathway regulation and how they systematically govern the effects of drugs on the body ${ }^{2}$ and play an important role in the treatment of many types of diseases. At the same time, TCM has become one of the new sources of drugs that can be used for many cardiovascular diseases including AMI because of their low side effects and satisfactory pharmacodynamic activity.

The lily, Tricyrtis maculata (D.Don) Machride, mainly distributed in northwest China, is from the Liliaceae family, and the entire plant has been used as an important folk medicine for the treatment of bruises, cough, tuberculosis, and ischemic cerebrovascular disease. In a previous study, we found that the extract of T. maculata can significantly improve microcirculation, inhibit thrombosis, and remove blood stasis. ${ }^{3}$ Few studies have reported its chemical composition and biological activity, and thus, the specific pharmacological mechanism of action is still unclear.

Network pharmacology is a new discipline that reveals the effect of TCM on the organism regulation network at the systematic level. Its systematic and holistic research concept is consistent with pluralistic, multieffect, and synergistic of TCM. ${ }^{4}$ Traditional network pharmacology, however, tends to focus on a single Chinese medicinal material or compound, neglecting the extraction of functional components with active roles. Therefore, basic research on effective substances is insufficient, and functional components of TCM require further elucidation.

To obtain additional data, we used a comprehensive research method that combines network pharmacology and experimental pharmacology to reveal the role and molecular mechanism of the functional components of T. maculata in the treatment of AMI. The basis of the active substance was determined, and the activity of active ingredients was verified (Figure 1), which provided theoretical support for the future research and development of new drugs.

\section{Network Pharmacology Research Databases}

The China National Knowledge Infrastructure (CNKI) (https://www.cnki.net/), Traditional Chinese Medicine Systems Pharmacology Database and Analysis Platform (TCMSP) (http://tcmspw.com/tcmsp.php), metaTarFisher database (https://metatarget.scbdd.com/home/index/), PubChem database (https://pubchem.ncbi.nlm.nih.gov), Chemical Source Network (https://www.chemsrc.com/), SwissTargetPrediction database, UniProt database (https://www.uniprot.org/), Cytoscape 3.7.2 software (http://www.cytoscape.org), Genecards database (https:// www.genecards.org), OMIM database (https://omim.org/), GEO database (https://www.ncbi.nlm.nih.gov/geo/), String 


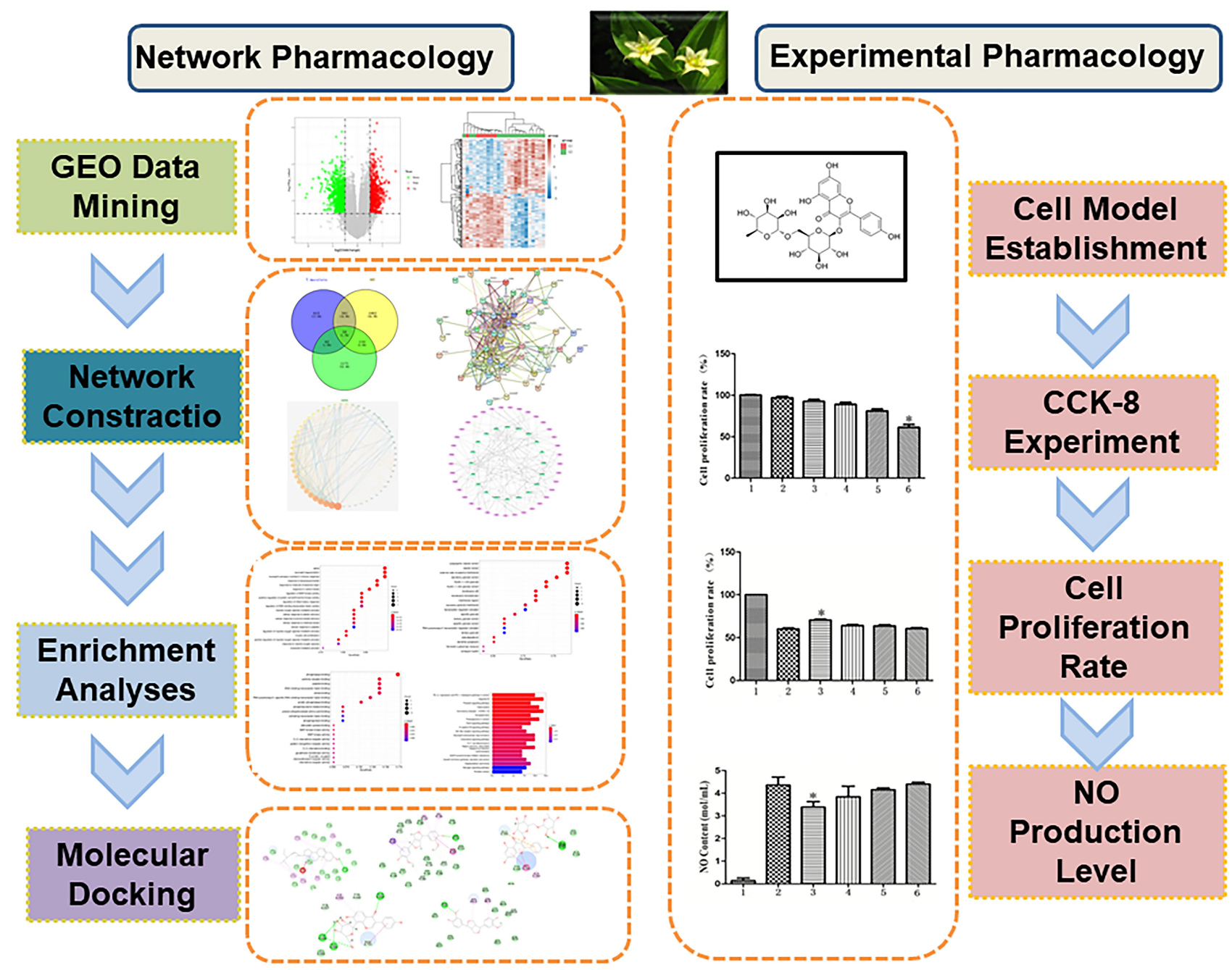

Figure I Graphical abstract.

database (https://string-db.org/), $\quad \mathrm{R} \quad$ language (ClusterProfiler package), Protein Data Bank (PDB) database (http://www1.rcsb.org/), and DrugBank database (https://go.drugbank.com/) were used.

\section{Collection of Drug Ingredients and Prediction of Targets of $T$. maculata}

Using data from our previous experiment, combined with literature reviewed through the China National Knowledge Infrastructure (CNKI), a total of 40 chemical constituents were obtained from T. maculata. ${ }^{5-9}$ The corresponding CAS numbers for all chemical constituents were obtained through the chemical source network, and then, the corresponding 2D structures and Simplified Molecular Input Line Entry System (SMILES) were obtained through the PubChem database. The chemical structures of all compounds were imported into the SwissTargetPrediction and metaTarFisher database to predict their potential targets. ${ }^{10}$

\section{Mining and Screening of AMI Disease Targets}

The GeneCards and OMIM database ${ }^{11,12}$ were used to screen disease targets. After entering "acute myocardial infarction" as the disease name in the search field, the targets related to AMI were collected and integrated. The results were output as an Excel spreadsheet. Through the intermediate value method, the correlation score method was proposed as a number of screening indexes, and the disease targets were obtained by deintegration method. Targets of differentially expressed genes from GEO database were obtained. The data from GSE60993 were downloaded and processed using the online analysis tool GEO2R. The criteria for gene screening 
were $\mathrm{P}<0.05$ and $|\log \mathrm{FC}| \geq 0.5$ to obtain the differentially expressed genes between AMI and normal tissues. The Heat Map package in $\mathrm{R}$ language was used to draw the heat map. ${ }^{13}$ Volcanic maps of differentially expressed genes were drawn based on multiple changes in gene expression in different experimental groups, such as abscissa, and the statistical significance of genome sequences.

\section{Cross Analysis of Wayne Drug and Disease Targets}

By using an online mapping tool (http://bioinformatics. psb.ugent.be/beg), the intersection of drug targets, disease targets and differential genes can be obtained.

\section{Construction of a Protein-Protein Interaction (PPI) Network}

The intersection gene was imported into the String database, the species was set up as a human, the protein interaction map was obtained, and the results were output in TSV format. Cytoscape software was used to study the topology of the protein interoperation network, and the protein targets in the top five were obtained. ${ }^{14}$

\section{Enrichment Analysis of Interselection Targets}

GO biological function enrichment analysis and KEGG pathway enrichment analysis were carried out on the 58 intersection targets obtained by $\mathrm{R}$ language, in which the GO biological function enrichment analysis included three parts, namely, GO biological processes (BPs), GO cellular components (CCs), and GO molecular functions (MFs) to find its possible pathway. ${ }^{15}$

\section{Construction of the "Key Components- Core Targets" Network}

Select the top 20 targets with the median value of the PPI network as the core targets, and the corresponding chemical components as the key components to construct "key components-core targets" network through Cytoscape (3.7.2) software, in which "node" represents the composition or target, and "edge" represents the interaction between nodes. The topological analysis of the target network was conducted using the "Analysis network" tool, in which the greater the degree value, the greater its role in the network. ${ }^{16}$

\section{Molecular Docking Verification}

Molecular docking was conducted between the active components of T. maculata and the core target to verify the interaction between them. First, the positive control drug of the corresponding target was found in the DrugBank database, and then, the 3D structure of the core protein, its corresponding ligand, and the 2D structure of the positive drug were downloaded from the PDB database as the control. The protein was dewatered by Discovery Studio 4.0 software, the binding site was found, the molecular docking was performed and scored in LibDock mode, and the matching score between the active component were compared and the positive control drugs. ${ }^{17}$

\section{Experimental Pharmacology Research \\ Experimental Material}

Human umbilical vein endothelial cell (HUVEC) lines were purchased from the China Center For Type Culture Collection, Dulbecco's modified Eagle's medium/Nutrient Mixture F-12 (DMEM/F-12) medium, penicillin, streptomycin, and fetal bovine serum (FBS) were purchased from Shanghai Beinuo Biotechnology Co., Ltd., and 0.25\% trypsin digestion solution was purchased from Sigma Company.

\section{Cell Culture Scheme and Grouping}

HUVECs were grown at $37^{\circ} \mathrm{C}$ in a $5 \% \mathrm{CO}_{2}$ incubator in a complete medium containing $20 \%$ FBS, $1 \%$ double antibody, and $80 \%$ Dulbecco's modified DMEM/F-12. The liquid was changed every two days and passaged at 1:3. Three groups were involved in this experiment: (i) control group, (ii) tumor necrosis factor (TNF)- $\alpha$ group, and (iii) TNF- $\alpha$ plus medicine group. The control group was cultured in HUVEC medium only, and the TNF- $\alpha$ group was cultured with $50 \mathrm{ng} \cdot \mathrm{mL}^{-1} \mathrm{TNF}-\alpha$ solution on the basis of the HUVEC culture. The TNF- $\alpha$ group was pretreated for 2 hours, and a compound solution was added at concentration of $1,0.1$, and $0.01 \mathrm{mg} \cdot \mathrm{mL}^{-1}$.

\section{Cell Counting Kit (CCK)-8 Assay for Cell Proliferation}

The HUVEC suspension $\left(1 \times 10^{6} \mathrm{~mL}^{-1}\right)$ was inoculated into a 96-well plate. After adherent growth, the supernatant was absorbed, and the cells were treated with 10 $\mu \mathrm{L}$ of different concentrations of compound solutions (20, 10,5 , and $\left.1 \mathrm{mg} \cdot \mathrm{mL}^{-1}\right)$. There were 6 wells for each group, and after incubation at $37^{\circ} \mathrm{C}$ for 4 hours, $10 \mu \mathrm{L}$ CCK-8 
reagent ${ }^{18}$ was added to each well. After 1 hour of culture, the absorbance at $450 \mathrm{~nm}$ was measured, to determine the cell proliferation rate.

\section{Protective Effect of Functional Components of $T$. maculata on Endothelial Cell Injury}

Griess reagents were prepared according to a previously published method. A series of sodium nitrite solutions (5, $10,20,40,60,100 \mathrm{mmoL} \cdot \mathrm{mL}^{-1}$ ) were transferred to 96 -well plates, with $50 \mu \mathrm{L}$ added to each well, and $50 \mu \mathrm{L}$ of reagents $\mathrm{A}$ and $\mathrm{B}$ were then added in turn. After adding the reagents, the 96-well plates were gently shaken for $10 \mathrm{~min}$ in an incubator at $37^{\circ} \mathrm{C}$. After the reaction was sufficient, the OD values of the samples in the 96-well plates were determined at $540 \mathrm{~nm}$. The standard curve for the determination of the nitric oxide (NO) content was obtained as follows: $\mathrm{Y}=0.06 \mathrm{X}$ $+0.0737, \mathrm{r}=0.9957$.

HUVEC suspensions $\left(1 \times 10^{6} \mathrm{~mL}^{-1}\right)$ were inoculated into a 96-well plate, and the supernatant was carefully absorbed after 24 hours of adherent growth, with $100 \mu \mathrm{L}$ serum-free medium in the control group and $90 \mu \mathrm{L}$ medium in the TNF$\alpha$ group. The TNF- $\alpha$ group was supplemented with a solution consisting of $80 \mu \mathrm{L}$ medium and $10 \mu \mathrm{L}$ compound (10, 5, 1, or $\left.0.5 \mathrm{mg} \cdot \mathrm{mL}^{-1}\right)$. Six wells were used for each concentration. After 2 hours of culture, $10 \mu \mathrm{L} \mathrm{TNF- \alpha}\left(50 \mathrm{ng} \cdot \mathrm{mL}^{-1}\right)$ was added to the TNF- $\alpha$ group and the TNF- $\alpha$ plus drug group. After 16-18 hours of culture, $50 \mu \mathrm{L}$ of supernatant was absorbed and moved to a new 96-well plate, and the amount of NO in the supernatant was calculated.

\section{Results}

\section{Acquisition Results of Drug Targets and Disease Targets}

The isolated chemical composition information from T. maculata is shown in Table 1. A total of 1116 predicted targets was obtained after de-reintegration of the data. Using the GenenCards and OMIM databases, 1655 disease targets were searched, and using the GEO database, 1425 differentially expressed genes were obtained between normal and AMI patients, including 682 upregulated genes and 743 downregulated genes. As can be seen from the volcano map (Figure 2A) and heat map (Figure 2B), there is a clear genetic difference between normal body tissues and those of sick patients. Combining the drug targets with the disease targets, 58 core targets were obtained by drawing Wayne diagrams online (Figure 3A).

\section{Results of Network Construction}

In the PPI network diagram, it can be seen that the 58 intersection targets have a relatively close interaction relationship (Figure 3B). The results of topological analysis directly reflect the size of the role of 58 intersection targets in network diagrams (Figure 3C). It can be seen from the "key components-core targets" network that T. maculata can play the role of anti-AMI effect through the overall synergistic action of multi-components and multi-targets (Figure 3D).

\section{Results of Biological Function and Pathway Enrichment Analysis of Targets}

GO biological function enrichment analysis of intersection targets was conducted using R language, and a total of 1206 GO items were identified, of which there were 1112 entries in biological process (Figure 4A). This mainly involves aging, response to lipopolysaccharide, response to molecule of bacterial origin, positive regulation of reactive oxygen species metabolic process, neutrophil degranulation, and regulation of reactive oxygen species metabolic process. There were 36 entries for the cellular component, including ficolin-1-rich granule, ficolin-1-rich granule lumen, cytoplasmic vesicle lumen, vesicle lumen, and external side of plasma membrane (Figure 4B), and there were 58 entries for molecular functional, including phosphatase binding, cytokine receptor binding, peptide binding, protein phosphatase binding, and adenylate cyclase binding (Figure 4C).

Based on the KEGG pathway enrichment analysis of the intersection targets, 127 items were identified (Figure 4D), namely, PD-L1 expression and PD-1 checkpoint pathway in cancer, Hepatitis B, Prolactin signaling pathway, Tuberculosis, Coronavirus disease - COVID-19.

\section{Results of Molecular Docking}

Five target proteins with the highest PPI value were selected as receptors, and corresponding chemical components were used as ligands for molecular docking to verify the binding activity between the active components of T. maculata and its potential targets against AMI.

The scores of corresponding compounds and positive control drugs in Table 2 were compared. MAPK3 and YD13, STAT3 and YD6, TLR4 and YD20, MAPK1 and YD20, MMP9 and YD21 exhibited satisfactory binding 
Table I 40 Known Chemical Compositions of T. maculata

\begin{tabular}{|c|c|c|c|}
\hline Numbering & Name & CAS Number & Reference \\
\hline YDI & 12 $\alpha$-hydroxyprogesterone & $19897-02-0$ & [6] \\
\hline YD2 & 3-O-Methylquercetin & $1486-70-0$ & {$[5]$} \\
\hline YD3 & N-benzoyl-L-phenylalaninol & $4503-96-2$ & [7] \\
\hline YD4 & Sitostenone. & $|058-6|-3$ & [6] \\
\hline YD5 & $\beta$-Carotene & $7235-40-7$ & [6] \\
\hline YD6 & Puerarin & $3681-99-0$ & [9] \\
\hline YD7 & Catechol & $120-80-9$ & [6] \\
\hline YD8 & 4-Methoxycinnamic acid & $830-09-1$ & [7] \\
\hline YD9 & $\beta$-Sitosterol & $83-46-5$ & {$[5]$} \\
\hline YDIO & Afzelin & $482-39-3$ & [6] \\
\hline YDII & Ferulic acid & II $35-24-6$ & [5] \\
\hline YDI2 & Methyl ferulate & $22329-76-6$ & [7] \\
\hline YDI3 & Stigmasterol & 83-48-7 & [6] \\
\hline YDI4 & 4-Hydroxybenzoic acid & $99-96-7$ & {$[5]$} \\
\hline YDI5 & Quercetin & $117-39-5$ & [5] \\
\hline YDI6 & Sinapaldehyde & $20649-43-8$ & [7] \\
\hline YDI7 & Rutin & $153-18-4$ & [9] \\
\hline YDI8 & Kaempferol & $520-18-3$ & [5] \\
\hline YDI9 & Coniferyl aldehyde & $20649-42-7$ & [7] \\
\hline YD20 & Pinoresinol & $487-36-5$ & [7] \\
\hline YD2I & Nicotiflorin & $17650-84-9$ & [6] \\
\hline YD22 & Indole-3-carboxaldehyde & $487-89-8$ & [7] \\
\hline YD23 & Protocatechuic acid & $99-50-3$ & [6] \\
\hline YD24 & Pregnenolone & $|45-13-|$ & [6] \\
\hline YD25 & Palmitic acid & $57-10-3$ & {$[5]$} \\
\hline YD26 & R-(-)-3-Hydroxy- $\beta$-ionone & $|540|-34-0$ & [8] \\
\hline YD27 & Methylparaben & $99-76-3$ & [8] \\
\hline \multirow[t]{2}{*}{ YD28 } & (S)-5-hydroxy-3,4-dimethyl & $6067-11-4$ & [8] \\
\hline & -5 -pentylfuran-2(5H)-one & & \\
\hline YD29 & Neoechinulin A & $5155 \mid-29-2$ & {$[8]$} \\
\hline YD30 & $5,7,2^{\prime}, 6^{\prime}$-tetrahydroxy dihydro flavonoids & - & [6] \\
\hline YD3। & $5 \alpha$-stigmastane- $3 \beta, 6 \beta$-diol & - & [6] \\
\hline YD32 & Triculata A & - & [7] \\
\hline YD33 & Trimethy-3,4-dehydrochebulate & - & [7] \\
\hline \multirow[t]{2}{*}{ YD34 } & Kaempferol-3-O- $\beta$-D-gluco & - & [6] \\
\hline & side-7-O- $\alpha$-L-rhamnoside & & \\
\hline YD35 & Balanophonin B & - & [8] \\
\hline YD36 & Ash resin alcohol & - & [8] \\
\hline YD37 & Syringaresinol & - & [8] \\
\hline YD38 & Lichenene & - & [8] \\
\hline YD39 & $4^{\prime}$-(I'-propenoic methylester) phenoxyl caffeate & - & [6] \\
\hline YD40 & Trimacoside A & - & [8] \\
\hline
\end{tabular}

Notes: The abbreviation of the Latin name of Tricyrtis maculata is as follows: T. maculata. 
A

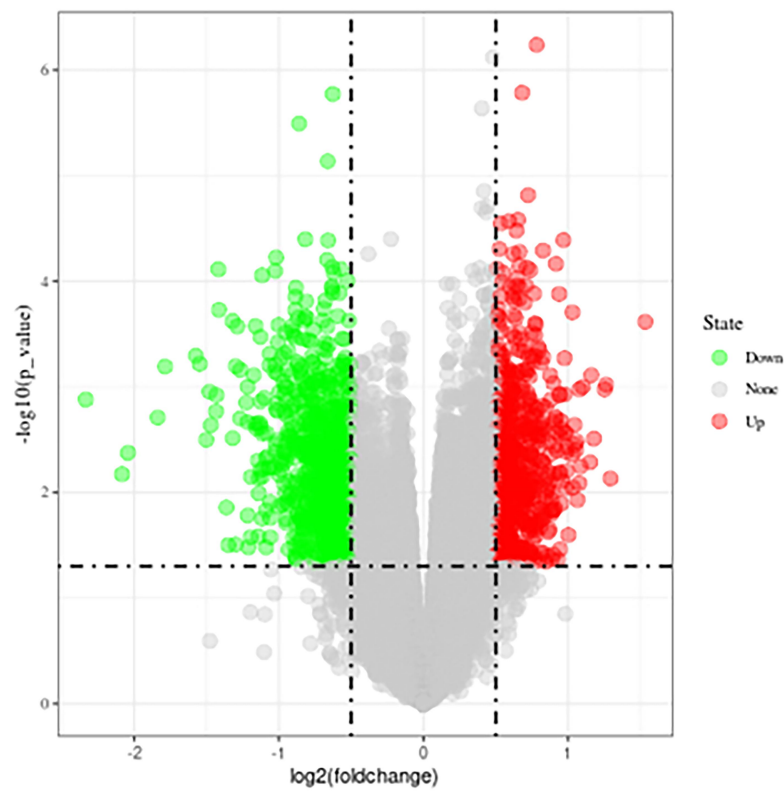

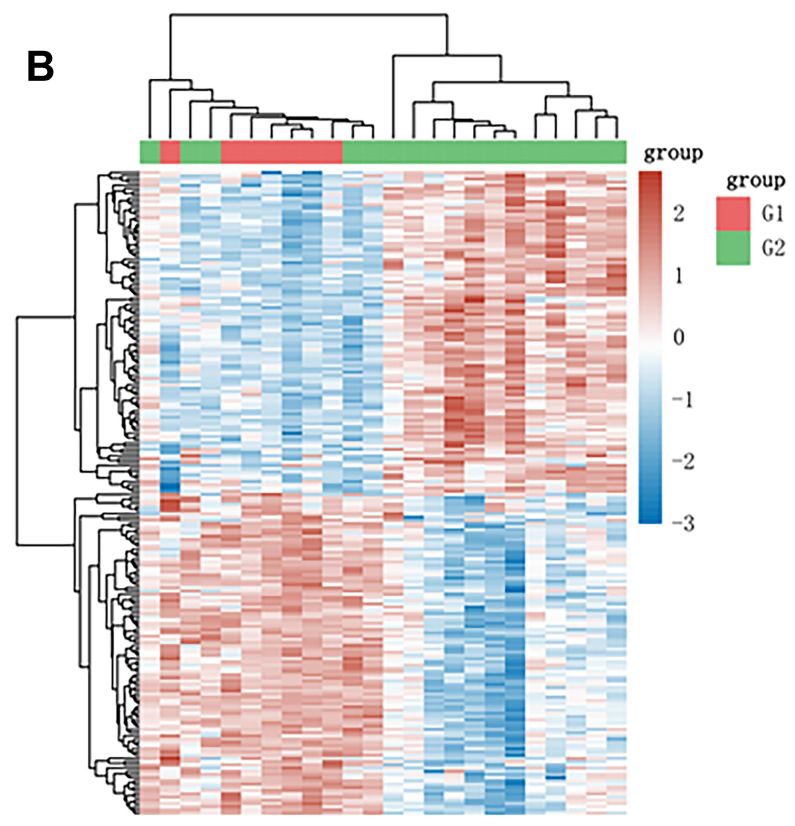

Figure 2 Results of GEO data mining. (A) Heat map of differential gene expression; (B) volcano map of differential gene expression.

activity, suggesting that these may be the core targets of anti-AMI action (Figure 5).

\section{Protective Effect of Nicotiflorin on HUVEC Damage \\ Effects of Different Concentrations of Nicotiflorin on HUVECS}

The effects of different concentrations of nicotiflorin on the growth and proliferation of HUVECs are shown in the Figure 6A. The results of the CCK-8 assay showed that nicotiflorin caused no significant toxic side effects on cells at a concentration of $0.05 \mathrm{mg} \cdot \mathrm{mL}^{-1}$, but cell activity decreased by $28 \%$ when the concentration reached $2 \mathrm{mg} \cdot \mathrm{mL}^{-1}$. Therefore, $0.05-1 \mathrm{mg} \cdot \mathrm{mL}^{-1}$ was selected as the optimal concentration in subsequent studies $(\mathrm{P}<0.05)$.

\section{Inhibitory Effect of Different Concentrations of Nicotiflorin on HUVEC Toxicity Induced by TNF- $\alpha$}

The effect of different concentrations of nicotiflorin on the proliferation of HUVECs is shown in Figure 6B. The results showed that the $24 \mathrm{~h}$ growth inhibition rate of HUVECs in response to $50 \mathrm{ng} \cdot \mathrm{mL}^{-1} \mathrm{TNF}-\alpha$ was $40 \%$, while the growth inhibition rates of HUVECs pretreated with $1,0.5,0.1$ and $0.05 \mathrm{mg} \cdot \mathrm{mL}^{-1}$ nicotiflorin were $21.84 \%, 34.78 \%, 36.61 \%$, and $39.77 \%$, respectively $(\mathrm{P}<0.05)$.
The effect of different concentrations of nicotiflorin on the NO production of HUVECs is shown in Figure 6C. The results show that the concentration of $\mathrm{NO}$ was 4.43 $\pm 0.54 \mathrm{ng} \cdot \mathrm{mL}^{-1}$ after treating HUVECs for $24 \mathrm{~h}$ with 50 $\mathrm{ng} \cdot \mathrm{mL}^{-1} \mathrm{TNF}-\alpha$, and the NO concentrations of HUVECs after treatment with $1,0.5,0.1$, and $0.05 \mathrm{mg} \cdot \mathrm{mL}^{-1}$ nicotiflorin were $3.29 \pm 0.47,4.00 \pm 0.60,4.12 \pm 0.23,4.30 \pm 0.16$ $\mathrm{ng} \cdot \mathrm{mL}^{-1}$, respectively. These research results show that nicotiflorin inhibits TNF- $\alpha$-induced NO production in a dose-dependent manner $(\mathrm{P}<0.05)$.

\section{Summary}

Based on the GEO data mining and molecular docking verification, we isolated monomer compounds with satisfactory anti-AMI activity. The molecular docking fraction showed that there was satisfactory binding activity between the functional components and the core target, with binding activity greater than that of existing drugs. Among them, nicotiflorin had a high binding score with the core target MMP 9, which ranks five in value, and as one of the relatively novel compounds in T. maculata, the content of total flavonoids in the effective part was as high as $1.075 \%$. It is speculated that nicotiflorin may be an active substance for the treatment of AMI, and on this basis, experiments were conducted in vitro.

HUVECs stimulated by TNF- $\alpha$ are mature inflammatory injury models. In the current study, TNF- $\alpha$ was used 


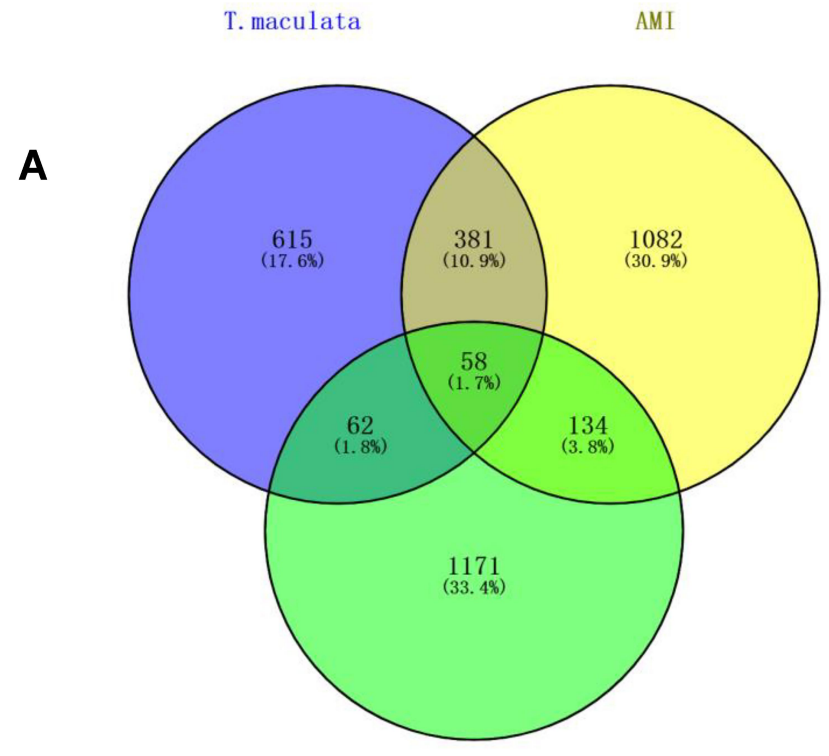

GE0

C

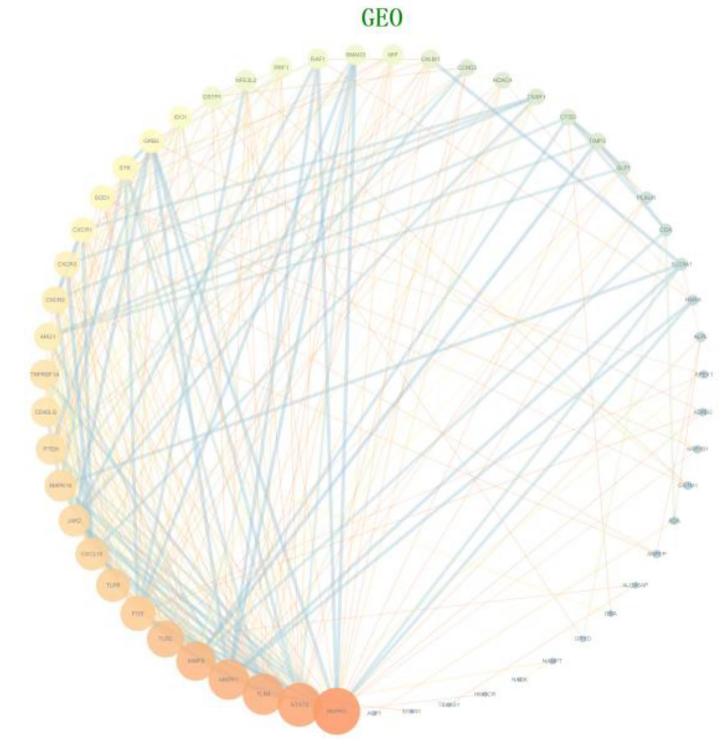

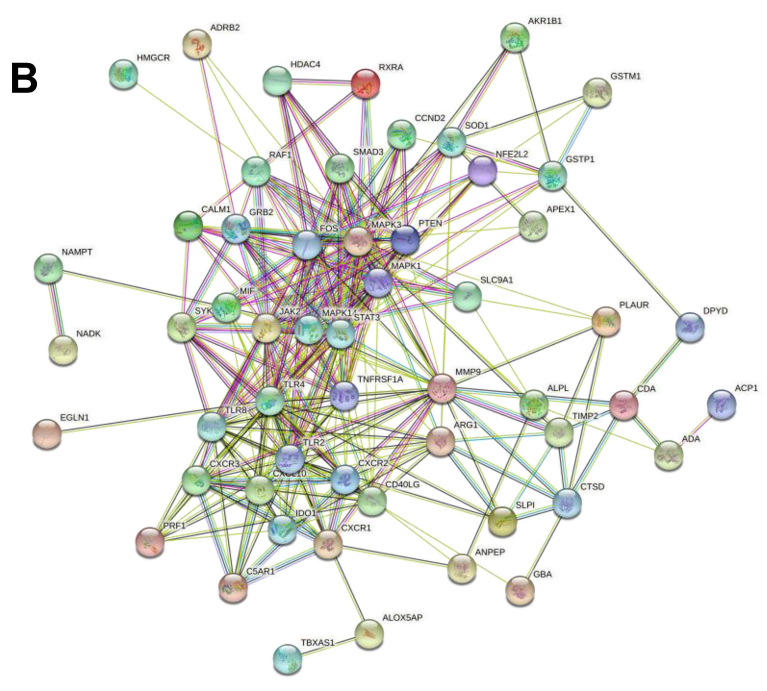

D

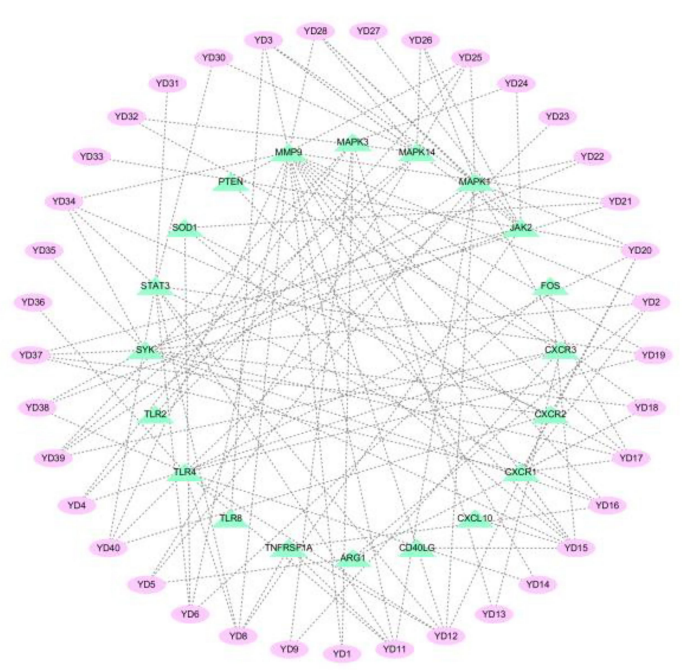

Figure 3 Results of network construction. (A) Venn diagram of component-disease intersection targets; (B) PPI network; (C) topological analysis results of PPI network; (D) results of "key components-core targets" network.

as an inducer ${ }^{19}$ to stimulate HUVECs to produce an inflammatory response. Herein, we explored how the different concentrations of nicotiflorin protect the endothelium in response to cell injury induced by TNF- $\alpha$, so as to lay a foundation for revealing the anti-inflammatory and anti-MI mechanisms of the functional components of T. maculata.

\section{Discussion}

The pathological basis of AMI is the rupture of atherosclerotic plaques in the coronary arteries, which leads to thrombosis and acute coronary occlusion. Unstable rupture of coronary plaque following atherosclerosis is caused by a variety of factors, such as physiological and psychological stress. If platelets accumulate on the surface of a ruptured atherosclerotic mass, this will result in a lack of blood flow to the heart, leading to continued severe myocardial ischemia and hypoxic necrosis. In addition, the pathogenesis of AMI is more complicated, involving oxidative stress, inflammatory damage, genetics, the environment and other factors.

Among them, oxidative stress and inflammatory damage are considered to be important links in the pathological process of AMI. According to previous studies, the myocardium of patients with AMI is in a state of excessive oxidative 
A

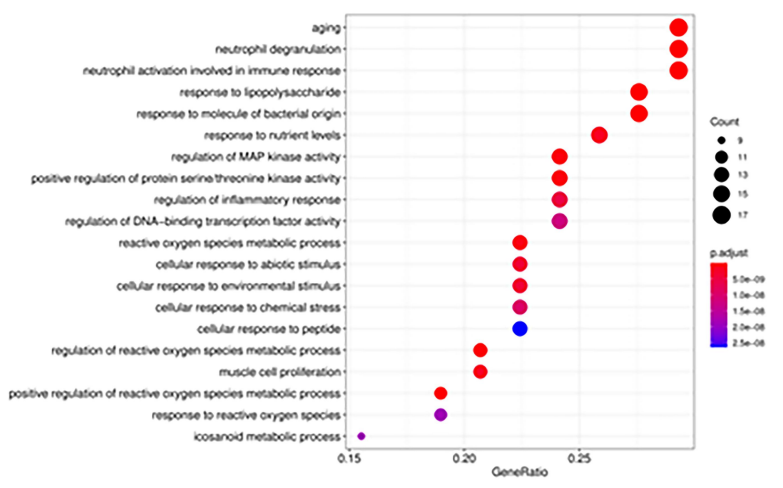

C

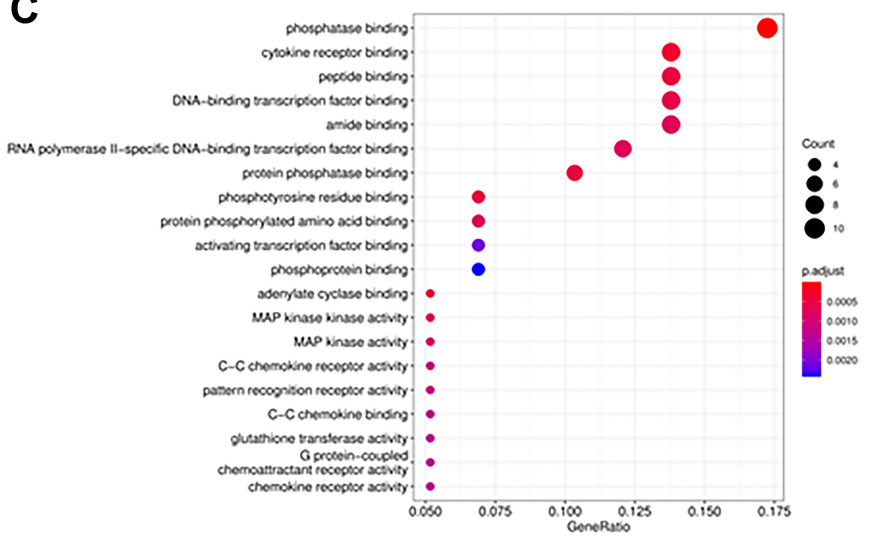

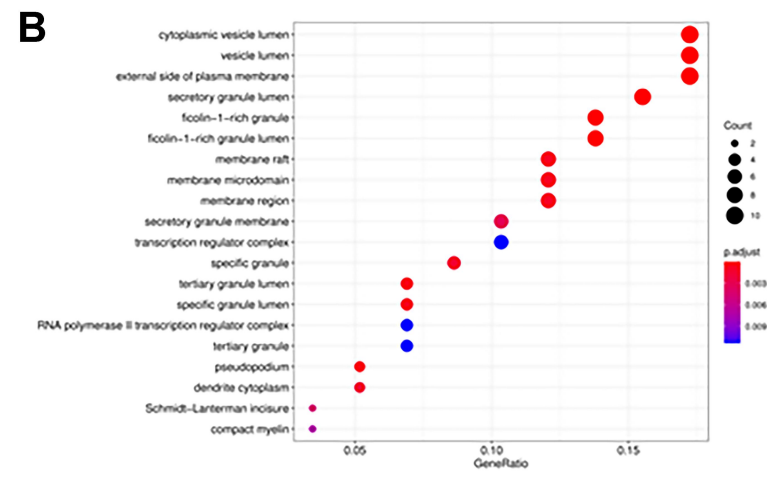

D

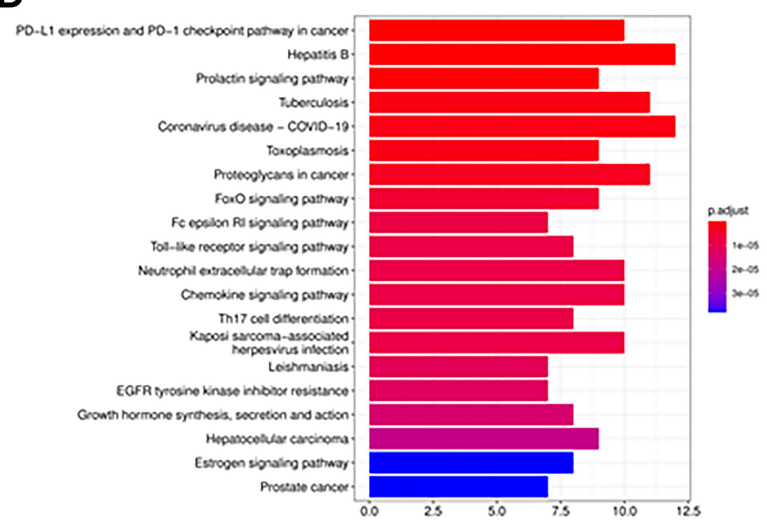

Figure 4 Results of GO and KEGG enrichment analysis. (A) GO BP enrichment analysis of interselection targets; (B) GO CC enrichment analysis of interselection targets; (C) GO MF enrichment analysis of interselection targets; (D) KEGG enrichment analysis of interselection targets.

stress. ${ }^{20}$ Excessive oxidative stress can damage vascular endothelial cells, reduce the stability of atherosclerosis, and accelerate thrombus formation. ${ }^{21}$ The inflammatory response occurs during atherosclerotic plaque formation, rupture, and myocardial ischemia-reperfusion injury after AMI. The current study confirmed that the amount of typical inflammatory factor TNF- $\alpha$ produced during myocardial reperfusion injury was positively correlated with the level of the cardiac functional index, that is, the level of inflammatory factors directly reflects the degree of myocardium reperfusion injury. ${ }^{22}$ In addition, TNF- $\alpha$ and IL-1 $\beta$ can also simultaneously inhibit smooth muscle cell collagen secretion, accelerate the dissolution of fibrous cap collagen, cause the instability of atherosclerotic plaque, and accelerate the process of $\mathrm{AMI}^{23}$

Nicotiflorin is a flavonoid glycoside extracted from T. maculata. Modern pharmacological studies show that nicotiflorin has anti-inflammatory, antioxidant, antibacterial, antiviral, analgesic, and neuroprotective effects. ${ }^{24-26} \mathrm{In}$ our previous studies, it also exhibited a good bloodactivating effect. In the rat model of permanent focal ischemia induced by $\mathrm{Li}$ et al, nicotiflorin decreased the infarcted area and behavioral defects induced by permanent focal cerebral ischemia (pMCAO). ${ }^{25}$ At the cellular level, nicotiflorin was able to upregulate the expression of mRNA and protein by enhancing the catalytic activity of endothelial nitric oxide synthase (eNOS) in endothelial cells. ${ }^{27}$ In the rat model of renal ischemia reperfusion C57BL, nicotiflorin decreased cell apoptosis in reperfusion injury by stably combining with ATF $3{ }^{24}$ In addition, it has been shown that nicotiflorin reduces the volume of infarction caused by ischemic injury and reduces oxidative stress and inflammatory response in rats with multiple dementia. ${ }^{28}$ However, there has no research describing the effect of nicotiflorin on cardiovascular ischemic injury.

The current study used network pharmacology to predict the possible mechanism of effective components in the treatment of AMI, and proves that nicotiflorin binds to the core target by molecular docking. The results of subsequent in vitro experiments show that it can significantly decrease the release of NO in a TNF- $\alpha$-induced HUVEC model and reduce the inflammatory response after cell injury, which initially proves that the endothelial protective effect is satisfactory. 
Table 2 Docking Scores of Target Protein and Its Corresponding Compound

\begin{tabular}{|l|c|c|c|c|}
\hline Target & Compound & Score & Positive Drug & Score \\
\hline MAPK3 & YD13 & 135.833 & Sulindac & 142.302 \\
STAT3 & YD6 & 102.054 & 16-tetraene-3-carboxamide & 89.0439 \\
TLR4 & YD20 & 94.7019 & Mifamurtide sodium hydrate & 21.6182 \\
MAPKI & YD20 & 133.44 & Isoproterenol & 102.414 \\
MMP9 & YD21 & 135.835 & Minocycline & 122.449 \\
\hline
\end{tabular}

Abbreviations: AMI, acute myocardial infarction; TCMSP, Traditional Chinese Medicine Systems Pharmacology Database and Analysis Platform; OMIM, Online Mendelian Inheritance in Man; PPI, protein-protein interaction; GO, Gene Ontology; BP, biological process; CC, cellular component; MF, molecular function; KEGG, Kyoto Encyclopedia of Genes and Genomes; NO, nitric oxide; AKT, protein kinase B; FoxO, forkhead box O; Bcl, B-cell lymphoma; TCM, Traditional Chinese Medicine; PDB, Protein Data Bank; CAS, Chemical Abstracts Service; HUVEC, human umbilical vein endothelial cell; FBS, fetal bovine serum; DMEM/F-I2, Dulbecco's modified Eagle's medium/nutrient mixture F-I2; CCK, cell counting kit; OD, optical density; MAPK, mitogen-activated protein kinase; pMCAO, permanent focal cerebral ischemia; eNOS, endothelial nitric oxide synthase; STAT, signal transducer and activator of transcription; TLR, toll-like receptors; MMP, matrix metalloproteinase; TNF- $\alpha$, tumor necrosis factor alpha; SOD, superoxide dismutase; ROS, reactive oxygen species.

According to the literature, many components such as quercetin, kaempferol, and $\beta$-sitosterol exhibit antiinflammatory and antioxidant activities, inhibit platelet aggregation, protect cardio-cerebral vessels, and reduce apoptosis and other pharmacological activities. ${ }^{29-32}$ The PPI network showed that MAPK3, STAT3, TLR4, MAPK1 and MMP9 possess large degrees of freedom, suggesting that they may be key targets of anti-AMI activity. MAPK1 and MAPK3 are mitogen activated protein kinases 1,3, which are involved in regulating stress, inflammation, cell cycle and other physiological activities. It was found that activation of p38 MAPK signaling pathway could reduce myocardial ischemia reperfusion injury in rats with heart failure. ${ }^{33}$ STAT3 is signal transduction and transcription activator 3 , which participates in cell growth and apoptosis, inhibits autophagy and regulates insulin secretion in macrophages. There is evidence that STAT3 activation protects the heart muscle from ischemiareperfusion injury, ${ }^{34}$ After STAT3 knockout, the cardiac development and function of mice were affected, and the cardiac injury was aggravated under pathological condition. $^{35}$ Toll-like receptor 4 (TLR4) is highly expressed in cardiomyocytes, which can mediate inflammatory response through many pathways, induce the release of a large number of inflammatory factors such as TNF- $\alpha$, IL- $1 \beta$ and IL-6, and aggravate cardiomyocyte injury. ${ }^{36}$ MMP-9 belongs to proteolytic enzyme, is an important part of extracellular matrix metalloproteinases family, is an important cause of atherosclerosis, plays a role in triggering thrombus, not only can degrade extracellular lipids, but also plays an important role in the migration and proliferation of vascular smooth muscle. It is a hot target of cardiovascular disease research in recent years. It was found that MMP9 could decrease the stability of plaque, increase the expression of MMP9 in patients with atherosclerosis. ${ }^{37}$

In the enrichment results of KEGG channels, after eliminating the first few channels that are not directly related to AMI, we focused on the FoxO signal paths, which rank eighth. FoxO transcription factors belong to the forkhead family of transcriptional regulation. It has been previously reported that the FoxO family participates in regulating many physiological activities, including inflammation, oxidative stress, apoptosis, glucose and lipid metabolism disorders, cyclic control, mitochondrial dysfunction and life span. ${ }^{38,39}$ The FoxO family is essential for maintaining heart homeostasis and regulating the size of cardiac myocytes, in addition to delaying the development of cardiovascular disease by increasing the antioxidant capacity of the cells. ${ }^{40}$

As two ubiquitous subtypes of cardiac myocytes in the family of FoxO transcription factors, FoxO1 and FoxO3 have been shown to be involved in regulating myocardial antioxidant stress and antiapoptosis. ${ }^{41}$ The up-regulated expression of both can inhibit the production of ROS, regulate the antioxidant gene SOD2 and the peroxidase Cat, enhance the anti-oxidative stress ability, inhibit cardiac myocyte apoptosis, and thus exert myocardial protective function. Cardiac myocyte-specific FoxO deficiency mice showed increased oxidative stress, apoptosis, and decreased cardiac function. Conversely, in cardiac myocytes with enhanced FoxO function, ROS production decreased, the expression of antioxidants and anti-apoptotic proteins increased, and apoptosis was inhibited. $^{40}$ In addition, AKT, as the upstream target of FoxO, plays an important role in regulating apoptosis. ${ }^{42}$ Activation of AKT can promote FoxO phosphorylation and cytoplasmic degradation, reduce the expression of 

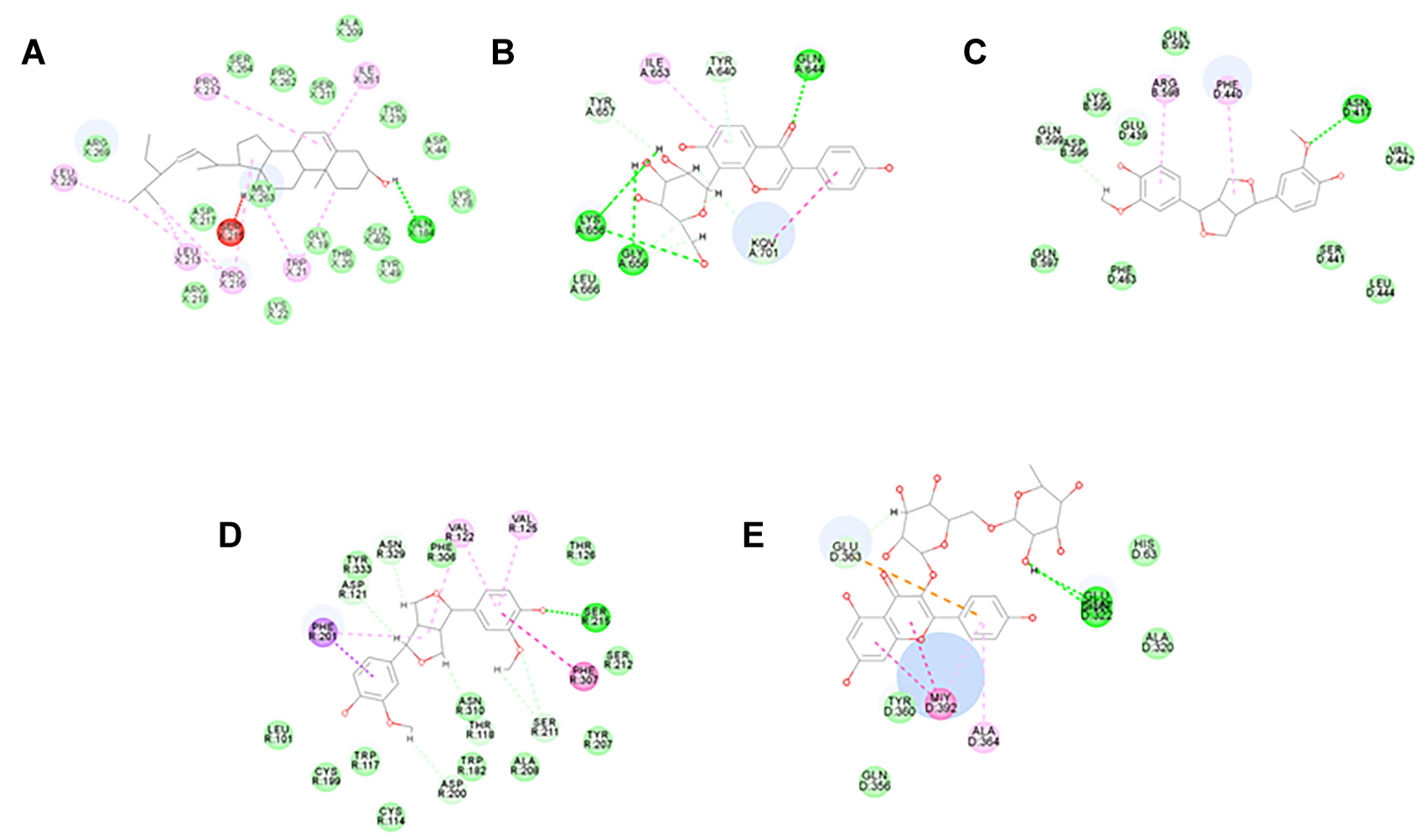

Figure 5 Molecular docking results. (A) MAPK3 and YDI3 molecular docking; (B) STAT3 and YD6 molecular docking; (C) TLR4 and YD20 molecular docking; (D) MAPKI and YD20 molecular docking; (E) MMP9 and YD2I molecular docking.

A

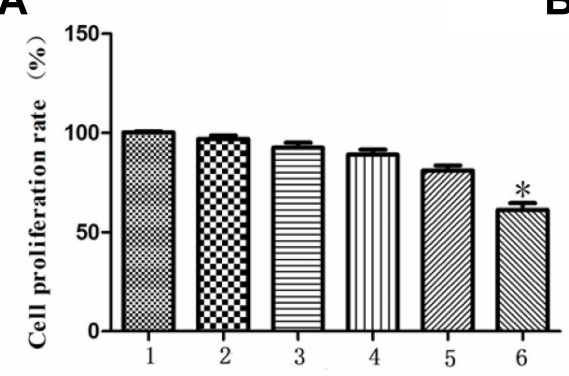

B

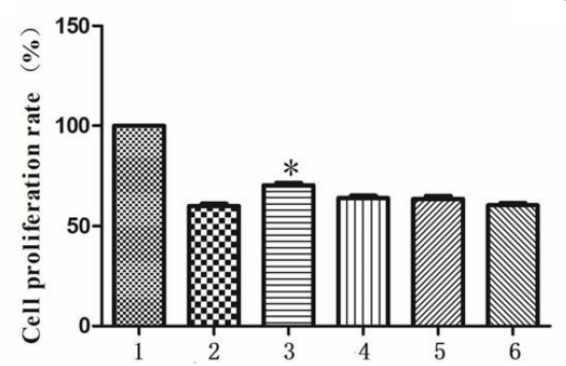

C

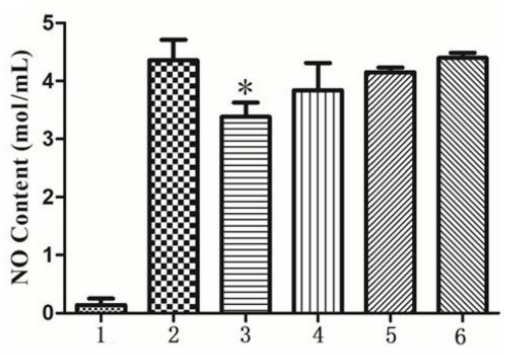

Figure 6 Results of the protective effect of nicotiflorin on TNF- $\alpha$-induced HUVEC damage. (A) Inhibition rate of HUVEC growth by different concentrations of nicotiflorin; (B) effect of different concentrations of nicotiflorin on the growth inhibition rate of HUVEC cells induced by TNF- $\alpha$; (C) comparison of NO level produced by nicotiflorin on TNF- $\alpha$ induced HUVEC cells supernatant $(* P<0.05)(n=5)$.

non-phosphated FoxO1 and FoxO3 in the cell nucleus, and improve the activity of anti-apoptotic protein $\mathrm{BCl}$, which can alleviate cardiac myocyte apoptosis. ${ }^{43}$

The network pharmacology and GEO data mining conducted in this study indicate that $T$. maculata may play a therapeutic effect on AMI by improving the transduction of $\mathrm{AKT} / \mathrm{FoxO} / \mathrm{BCl}$ signaling pathway. During the research process we conducted, nicotiflorin, a monomeric pyroside with satisfactory activity, was unexpectedly discovered. To verify the accuracy of the network pharmacological results, an experimental pharmacological study of pyroside was conducted by constructing a TNF- $\alpha$-induced HUVEC endothelial injury model to verify whether it has a preprotective effect on endothelial cell injury.

In vitro cell experiments showed that nicotiflorin inhibited the release of inflammatory mediator $\mathrm{NO}$ after endothelial cell injury and protected against endothelial cell injury. From the point of view of network pharmacology and molecular docking, it is speculated that the antiAMI mechanism may be related to the regulation of the 
$\mathrm{Akt} / \mathrm{FoxO} / \mathrm{BCl}$ signaling pathway. Akt activates the phosphorylation of lower transcription factor FoxO1, weakens its transcriptional function, decreases the activity of apoptotic protein bax in myocardium, reduces apoptosis, and enhances the tolerance of cardiomyocytes to acute ischemic injury. At the same time, FoxO is involved in the anti-oxidative stress process after myocardial ischemia. By reducing the production of free radicals, it can reduce the oxidation damage, improve the survival rate of cardiac myocytes and maintain the normal physiological function of myocardium.

\section{Conclusion}

This study elaborated the role of T. maculata in AMI and its potential molecular mechanisms. Using network pharmacology and GEO data mining, the potential protein targets and mechanisms of the components in T. maculata in the treatment of AMI were preliminarily predicted. In addition, molecular docking technology was used to verify the binding activity between the functional components and the core target. The results of network pharmacology indicated that T. maculata had an effect on $\mathrm{AMI}$ by modulating the $\mathrm{Akt} / \mathrm{FoxO} / \mathrm{BCl}$ signaling pathway. It is speculated that the anti-AMI effect of T. maculata may be related to cyclic regulation, reduce inflammatory response, myocardial cell apoptosis inhibition, and energy metabolism regulation. Through the extraction and experimental verification of active components from medicinal plants, the monomer named nicotiflorin was obtained, and it demonstrated satisfactory activity. By establishing a TNF-HUVEC damage model, the anti-inflammatory effect and endothelial protection of nicotiflorin was verified in vitro. Nicotiflorin may be a compound that can potentially be used as a new anti-AMI drug with excellent prospects for clinical application. Our study lays a theoretical foundation for further research and development of new drugs.

\section{Author Contributions}

All authors contributed to data analysis, drafting or revising the article, have agreed on the journal to which the article will be submitted, gave final approval of the version to be published, and agree to be accountable for all aspects of the work.

\section{Funding}

This study is supported by the Shaanxi University of Traditional Chinese Medicine, the key technology innovation team for the integration of traditional Chinese medicine (2018TD-005).

\section{Disclosure}

The authors declare that they have no conflicts of interest in this work.

\section{References}

1. Ge Y, Li R, Yang Q, Li N, Zhao X. Experimental study on the intervention of Yiqi Huoxue recipe on MAPKs signaling pathway in acute myocardial infarction. Chin Arch Trad Chin Med. 2020;1-12.

2. Xie J, Gao S, Li L, Xu Y, Gao S, Yu C. Research progress and application strategy on network pharmacology in Chinese materia medica. Chin Trad Herbal Drugs. 2019;50(10):2257-2265. doi:10.7501/j.issn.0253-2670.2019.10.001

3. Sun J, Jia T. Preliminary study on the material basis of the activating blood effect of Tricyrtis maculata. J Chin Med Mater. 2016;39 (02):419-421. doi:10.13863/j.issn1001-4454.2016.02.045

4. Hopkins A. Network pharmacology. Nat Biotechnol. 2007;25 (10):1110-1111. doi:10.1038/nbt1007-1110

5. Yang J, Zhang D, Zhang X, Sun J. Chemical constituents of Tricyrtis maculata (D. Don) Machride. Central South Pharm. 2017;15 (07):922-924. doi:10.7539/j.issn.1672-2981.2017.07.014

6. Yang J. Study on Chemical Constituents of Four Medicinal Plants of Taibai Mountain. Northwest A\&F University; 2011.

7. Ren L, Wang Y, Zhang W, et al. Tricyrtis maculataTriculata A, a novel compound from (D. Don) J. F. Macbr. with biological properties. Nat Prod Res. 2020;1-9. doi:10.1080/ 14786419.2020.1736059.

8. Wang Y, Zhang W, Ren L, Sun J, Zhang D. Trimacoside A, a high molecular weight antioxidant phenylpropanoid glycoside from Tricyrtis maculata. Records Nat Prod. 2021;15(3):194-201. doi:10.25135/rnp.209.20.09.1810

9. Ren L, Jia T, Li X, et al. Study on quality standard of Tricyrtis maculata. China Pharm. 2019;30(08):1083-1090. doi:10.6039/j. issn.1001-0408.2019.08.14

10. Daina A, Michielin O, Zoete V. SwissTargetPrediction: updated data and new features for efficient prediction of protein targets of small molecules. Nucleic Acids Res. 2019;47(W1):W357-W364. doi:10.1093/nar/gkz382

11. Wu Y, Wang Y, Du Z, Li J, Liu Q, Shi Y. Study on network pharmacology of water-soluble components of salvia miltiorrhiza in treating coronary heart disease. J Chongqing Univ Technol. 2020;1-12.

12. Li L, Chen J, Guo Y, Han T. Research on the mechanism of TCM turmeric in treating depression based on network pharmacology. $J$ Liaoning Univ Trad Chin Med. 2020;22(02):121-125. doi:10.13194/j.issn.1673-842x.2020.02.033

13. Zhao Z, Hu S. A study on the mechanism of the Haizao Yuhu decoction regulating papillary thyroid cancer based on network pharmacology and molecular docking technology. Clin J Chin Med. 2020;12(29):1-8. doi:10.3969/j.issn.1674-7860.2020.29.001

14. Huang X, Yu G, Tong J. Analysis of the pharmacological mechanism of tangerine peel based on network pharmacology. Chin Trad Patent Med. 2019;41(12):3038-3045. doi:10.3969/j.issn.1001-1528.2019.12.043

15. Jia Y, Zou J, Wang Y, et al. Action mechanism of Roman chamomile in the treatment of anxiety disorder based on network pharmacology. J Food Biochem. 2021;45(1):e13547. doi:10.1111/jfbc.13547

16. Chen Y, Liu C, He T, et al. Network pharmacology study on Danshen Decoction in treatment of diabetic cardiomyopathy. Chin Trad Herbal Drugs. 2019;50(05):1164-1174. doi:10.7501/j.issn.02532670.2019.05.021 
17. Song X, Zhou W, Chen C, Wang S, Liang S. Study on material base of Ligusticum wallichii for treating brain ischemia and its molecular mechanism based on molecular docking. China J Chin Materia Medica. 2015;40(11):2195-2198. doi:10.4268/cjcmm20151124

18. Fang Q, Zhang Y, Jiang D, Chen Y. Hydroxytyrosol inhibits apoptosis in ischemia/reperfusion-induced acute kidney injury via activating Sonic Hedgehog signaling pathway. Eur Rev Med Pharmacol Sci. 2020;24(23):12380-12388. doi:10.26355/eurrev_202012_24032

19. Xu S, Zhao Y, Yu L, Shen X, Ding F, Fu G. Rosiglitazone attenuates endothelial progenitor cell apoptosis induced by TNF- $\alpha$ via ERK/

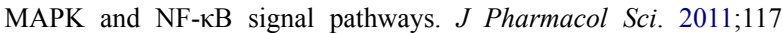
(4):265-274. doi:10.1254/jphs.11149fp

20. Li B, Tian J, Sun Y, et al. Activation of NADPH oxidase mediates increased endoplasmic reticulum stress and left ventricular remodeling after myocardial infarction in rabbits. Biochim Biophys Acta. 2015;1852(5):805-815. doi:10.1016/j.bbadis.2015.01.010

21. Wang S. Clinical effect of Shexiangbaoxin pills combined with fasudil on acute myocardial infarction and impact on micro-inflammatory state and vascular endothelial function. Pract $J$ Cardiac Cerebral Pneumal Vasc Dis. 2016;24(03):84-86. doi:10.3969/j.issn.1008-5971.2016.03.024

22. Cheng X, Liao Y, Li B, et al. Effects of early treatment with metoprolol on myocardial inflammatory cytokine expression and heart function in rats with acute myocardial infarction. Chin J Cardiol. 2005;33(5):448-452.

23. Fan J, Li J, Zhu D. Clinical diagnostic significance of serum Mb, cTnI and inflammatory factors IL-1 $\beta$ and TNF- $\alpha$ in patients with acute myocardial infarction. Exp Lab Med. 2017;35(05):724-727. doi:10.3969/j.issn.1674-1129.2017.05.032

24. Wang L, Li C, Guan C, et al. Nicotiflorin attenuates cell apoptosis in renal ischemia-reperfusion injury through activating transcription factor 3. Nephrology. 2021;26(4):358-368. doi:10.1111/nep.13841

25. Li R, Guo M, Zhang G, Xu X, Li Q. Neuroprotection of nicotiflorin in permanent focal cerebral ischemia and in neuronal cultures. Biol Pharm Bull. 2006;29(9):1868-1872. doi:10.1248/bpb.29.1868

26. Jia T, Guo T, Tian M, Zhai S, Yang Q, Sun J. LC-MS/MS determination of distribution of nicotiflorin in rats and its excreta. Pharmacol Clin Chin Materia Medica. 2020;36(06):69-76. doi:10.13412/j.cnki. zyyl.2020.06.007

27. Li R, Guo M, Zhang G, Xu X, Li Q. Nicotiflorin reduces cerebral ischemic damage and upregulates endothelial nitric oxide synthase in primarily cultured rat cerebral blood vessel endothelial cells. J Ethnopharmacol. 2006;107(1):143-150. doi:10.1016/j. jep.2006.04.024

28. Huang J, Fu S, Jiang Y, et al. Protective effects of Nicotiflorin on reducing memory dysfunction, energy metabolism failure and oxidative stress in multi-infarct dementia model rats. Pharmacol Biochem Behav. 2007;86(4):741-748. doi:10.1016/j.pbb.2007.03.003

29. Yang Y, Zeng X, Lei G. Quercetin protects cardiomyocytes subjected to anoxia/reoxygenation injury. Med Forum. 2019;23(17):2377-2379. doi:10.19435/j.1672-1721.2019.17.005

30. Lin W, Wang W, Wang D, Ling W. Quercetin protects against atherosclerosis by inhibiting dendritic cell activation. Mol Nutr Food Res. 2017;61(9):1700031. doi:10.1002/mnfr.201700031
31. Wang C, Guo C, Li X, et al. Protective effects of Kaempferol on hypoxic injury of myocardial cells through activation of mTOR pathway. Chin J Immunol. 2019;35(07):781-785. doi:10.3969/j. issn.1000-484X.2019.07.003

32. Yan N, Yang C, Ma J, et al. Effects of $\beta$-sitosterolon myocardial ischemia-reperfusion injury and ERK1/2 signaling pathway in rats. Adv Cardiovasc Dis. 2020;41(03):321-325. doi:10.16806/j.cnki. issn.1004-3934.2020.03.026

33. Zhang Y, Jin S, He S, Zhang S, Zhang Y, Chen L. Role of JNK and p38 MAPK signal pathways in morphine pretreatment to reduce myocardial ischemia reperfusion injury in heart failure rats. Chin $J$ Anesthesiol. 2016;36(02):219-222.

34. Oshima Y, Fujio Y, Nakanishi T, et al. STAT3 mediates cardioprotection against ischemia/reperfusion injury through metallothionein induction in the heart. Cardiovasc Res. 2005;65(2):428-435. doi:10.1016/j.cardiores.2004.10.021

35. Luo W, Huang L, Wang J, et al. Inhibition of EGFR-STAT3 attenuates cardiomyopathy in streptozotocin-induced type 1 diabetes. J Endocrinol. 2019;242(3):199-210. doi:10.1530/joe-19-0058

36. Cheng X, Yang Y, Yang H, Wang Y, Du G. Kaempferol alleviates LPS-induced neuroinflammation and BBB dysfunction in mice via inhibiting HMGB1 release and down-regulating TLR4/MyD88 pathway. Int Immunopharmacol. 2018;56:29-35. doi:10.1016/j. intimp.2018.01.002

37. Vatankulu M, Bacaksiz A, Sonmez O, et al. Does spironolactone have a dose-dependent effect on left ventricular remodeling in patients with preserved left ventricular function after an acute myocardial infarction? Cardiovasc Ther. 2013;31(4):224-229. doi:10.1111/ 1755-5922.12006

38. Hou Y, Yao Y, Bao Y, et al. Juglanthraquinone C induces intracellular ROS increase and apoptosis by activating the Akt/Foxo signal pathway in HCC cells. Oxid Med Cell Longev. 2016;2016:4941623. doi:10.1155/2016/4941623

39. Ning Y, Li Z, Qiu Z. FOXO1 silence aggravates oxidative stress-promoted apoptosis in cardiomyocytes by reducing autophagy. J Toxicol Sci. 2015;40(5):637-645. doi:10.2131/jts.40.637

40. Sengupta A, Molkentin J, Paik J, DePinho R, Yutzey K. FoxO transcription factors promote cardiomyocyte survival upon induction of oxidative stress. J Biol Chem. 2011;286(9):7468-7478. doi:10.1074/jbc.M110.179242

41. Chistiakov DA, Orekhov AN, Bobryshev YV. The impact of FOXO-1 to cardiac pathology in diabetes mellitus and diabetes-related metabolic abnormalities. Int J Cardiol. 2017;245.

42. Nakae J, Park B, Accili D. Insulin stimulates phosphorylation of the forkhead transcription factor FKHR on serine 253 through a Wortmannin-sensitive pathway. J Biol Chem. 1999;274 (23):15982-15985. doi:10.1074/jbc.274.23.15982

43. Cheng S, He X, Huang J, Yang P, Fan Z. Formononetin attenuates myocardial injury in diabetic mice through AKT/FoxO1 signaling. Trad Chin Drug Res Clin Pharmacol. 2020;31(10):1165-1172. doi:10.19378/j.issn.1003-9783.2020.10.006

\section{Publish your work in this journal}

Drug Design, Development and Therapy is an international, peerreviewed open-access journal that spans the spectrum of drug design and development through to clinical applications. Clinical outcomes, patient safety, and programs for the development and effective, safe, and sustained use of medicines are a feature of the journal, which has also been accepted for indexing on PubMed Central. The manuscript management system is completely online and includes a very quick and fair peer-review system, which is all easy to use. Visit http://www. dovepress.com/testimonials.php to read real quotes from published authors. 